\title{
BIODIVERSITY AND HEALING ACTIVITIES OF MEDICINAL PLANTS IN THE AREA OF KAMCHIA NATURE COMPLEX
}

\author{
Djeni Cherneva, Dobri Ivanov \\ Department of Biology, Faculty of Pharmacy, Medical University of Varna
}

\begin{abstract}
INTRODUCTION: Kamchia Nature Complex is part of the wetlands of importance in Bulgaria and has a global significance for preserving Europe's unique freshwater swamp forests also known as flooded (floodplain) forests (Bulgarian, Turkish: longoz).

AIM: The study aims to bring up to date the list of medicinal plants in the area of Kamchia Nature Complex and to create a present-day database of their ecological and biological characteristics, floral elements, conservation significance and status. In addition, this study is designed to collect data available on the healing properties, usable parts, and the groups of diseases these medicinal plants are applicable for.
\end{abstract}

MATERIALS AND METHODS: Field surveys were conducted during the 2013-2015 vegetation seasons applying enroute survey methods.

Floristic analysis was performed by the Tolmachev's method (1974).

Species were determined by "Flora of the Republic of Bulgaria" and "Identification. Guide to Higher Plants in Bulgaria".

RESULTS AND DISCUSSION: We have identified 183 species of medicinal plants out of 435 species of higher plants. The established medicinal plants refer to 60 families and 150 genera. The prevailing biology type is the herbaceous perennial plants-102 species (56\%). The mesophyte plants occupy dominant position in terms of moisture and humidity as a factor -91 species $(50 \%)$.

Floristic analysis reveals Eurasian geo-elements as being predominant-34 (19\%), with 60 (33\%) species of different types of Mediterranean distribution.

Species of conservation significance represent $20 \%$ of medicinal plants.

The established medicinal plants have more than 30 species of healing activities, one third of which is used primarily for treatment of gastrointestinal and respiratory diseases. Species in which the above ground por-

tion of the plant is collected for its plant substance constitute half of the established medicinal plants.

Address for correspondence:

Djeni Cherneva

Faculty of Pharmacy

Medical University of Varna

84 Tzar Osvoboditel Blvd

9002 Varna

e-mail:djeni_cherneva@abv.bg

CONCLUSION: Survey results reveal a considerable variety of medicinal plants in Kamchia Natural Complex area. They feature a variety of healing properties and are applicable for a wide range of diseases.

Keywords: wetlands, medicinal plants, Kamchia

Received: November 7, 2020

Nature Complex

Accepted: December 15, 2020 


\section{INTRODUCTION}

Kamchia Nature Complex is part of the wetlands of importance in Bulgaria and has a global significance for preserving Europe's unique freshwater swamp forests also known as flooded (floodplain) forests (Bulgarian, Turkish: longoz) (1). Kamchia river floodplain around the river's estuary and its downstream are in the Red List critically endangered category wetlands in Bulgaria with Identification No. 0985; Kamchia River estuary and Maznia Azmak flooded area are in the vulnerable wetlands category with Identification No. 9010 and No. 0758 (2).

Intensive floristic studies of Kamchia Nature Complex area were carried out within the 1992-1994 period as part of the North Wetlands Coastal Area project of the Bulgarian-Swiss Biodiversity Conservation Programme (BSBCP) (3). However, there is limited evidence and few publications on the biological diversity of medicinal plants in the area $(3,4,5)$.

\section{AIM}

The present study is part of a larger survey of the biological diversity of medicinal plants of the Northern Black Sea wetlands in Bulgaria and aims to supplement the available research data on medicinal plants in the Kamchia Nature Complex. We take aim to create an up-to-date database of their ecological and biological characteristics, floral elements, conservation significance and status. Along with it we collected data on the healing activities and usable parts of the established medicinal plants, together with data on the diseases they are applicable for.

\section{MATERIALS AND METHODS}

The surveyed territory is located approx. 20-25 $\mathrm{km}$ south of Varna and stretches east of the VarnaBurgess route to the village of Staro Oryahovo. It includes floodplain forests known as "Bulgarian Longoz, extensive areas of sandy dunes and a beach strip, shrub and grasslands, freshwater marshes and marine aquaria, as well as adjacent fishponds" (3).

Field surveys were conducted during the 20132015 vegetation seasons applying enroute survey methods.

Floristic analysis was performed by the Tolmachev's method (1974) (6).
Species were determined by "Flora of the Republic of Bulgaria" $(7,8,9,10,11)$ and "Identification. Guide to Higher Plants in Bulgaria” (12).

Florigenic analysis of the species was done according to the classification of Asyov and Petrova (2006) (13).

Status of medicinal plants was determined by the Medicinal Plants Act $(2000,2014)(14)$ and the National Strategy for Biodiversity Conservation (15).

The conservation status of species was defined at national level according to the "Red Data Book of Bulgaria" (16), the Biological Diversity Act (2002, 2007) (17), Order RD-83 of 03.02.2014 (18), and at an international level as defined in Lucas (1983) (19), the IUCN Red List (2014) (20), Appendix 1 to the Convention on the Conservation of European Wildlife and Natural Habitats (21) and the Appendices to the Convention on International Trade in Endangered Species of Wild Fauna and Flora (22). Endemism was presented at the level of Balkan and Bulgarian endemics according to "Balkan Endemics in the Bulgarian Flora" (23) and "List of Bulgarian Endemic Plants" (24).

Phytotherapeutic properties of the plants were described as per Petkov (1982) (25), Asenov (1988) (26), and Nikolov (2006) (27); the applications in traditional medicine were according to Petkov (1982) (25).

\section{RESULTS AND DISCUSSION}

We identified 435 species of higher plants in the Kamchia Nature Complex area. We determined 183 species or $42 \%$ of the flora as medicinal plants. Of these, 173 (40\%) were medicinal plants as per the Medicinal Plants Act $(2000,2014)$ and 84 species $(19 \%)$ were classified as medicinal plants according to the National Strategy for Biodiversity (14, 15, Hardalova et al. 1994). They accounted for $42 \%$ of the area's higher flora community and $24 \%$ of the wild plants in Bulgaria.

The medicinal plants established for the area belonged to 60 families and 150 genera. Families with the greatest number of species were: Asteraceae-21 (11\%), Lamiaceae-13 (7\%), Fabaceae-12 (7\%), Rosaceae-11 (6\%), Apiaceae-10 (5\%), Ranunculaceae -7 (4\%), Scrophulariaceae-7 (4\%), and Polygonaceae-6 (3\%), accounting for $47 \%$ of the established species. There were 9 less represented families 
with 4 species each, 4 families with 3 species each, 10 families with 2 species, and 28 families were represented by 1 species each. Apart for Poaceae, Ranunculaceae, and Polygonaceae, the rest of the families were duly represented with regards to the number of species in the flora of surveyed area (3).

Herbaceous perennial type plants were the predominant plant biology type-102 species (56\%), followed by the annual ones-28 species (15\%), and the shrubs-19 species (10\%). Biennial plants were represented by 13 species (7\%), the typical tree species were $11(6 \%)$, annuals to biennials accounted for 6 species (3\%), while biennials to perennials and shrubs to trees had 2 species (1\%) to account for.

Analysis displayed that distribution of the established for the area medicinal plants by biological type followed the described flora species distribution. Herbaceous perennial plants were prevalent (241 species or 54\%), followed by the annuals (92 species-21\%) and the shrubs (24 species or 6\%) (3).

Considering moisture and humidity as a factor, Mesophyte plants were the dominant medicinal plants-91 species (50\%), followed by Hygrophytes-46 species (25\%), and Xerophytes-41 species $(22 \%)$. Hydrophytes were represented by 5 species (3\%) only. The flora of Kamchia Nature Complex had similar ecological structure with Mesophytes (225 species or 52\%) being the predominant type, followed by Hygrophites (109 species or 24\%) and xerophytes (89 species or 21\%). Hydrophytes were represented by 12 species or 3\% (3) only.

Primary florigenic analysis revealed prevalence of Eurasian geo-elements-34 (19\%). They were dominant in the flora of the surveyed area as well-119 species (26\%) (3). Euro-Mediterranean came next-28 (15\%), followed by Euro-Siberian-23 (13\%), European-17 (9\%), Cosmopolitans-16 (9\%), sub-Mediterranean-16 (9\%), boreal-13 (7\%), subboreal-13 (7\%), and others. Generally, there were 60 species with different types of Mediterranean distribution, thus accounting for $33 \%$ of the total number of medicinal species. Percentage representation of Mediterranean species-33\%, was similar for Kamchia Natural Complex.

Analyses suggested that $20 \%$ of the 183 established medicinal plants belonged to different conservation categories.
The European Red List for endangered species lists 18 species under the category of near threatened: Alisma plantago-aquatica L, Leucojum aestivum L., Angelica sylvestris L., Bidens tripartita L, Carpinus betulus L., Pulmonaria officinalis L., Myriophyllum spicatum L., Iris pseudacorus L., Lycopus europaeus L., Mentha aquatica L., Lemna minor L., Colchicum autumnale L., Lythrum salicaria L., Nymphaea alba L., Rumex hydrolapathum Huds., Lysimachia nummularia L., Samolus valerandi L., Caltha palustris L.

The Bulgarian Red Data Book for endangered species includes three species as endangered: Eryngium maritimum L., Nuphar lutea (L.) S. et .S., Nymphaea alba L. Protected plants are Eryngium maritimum L., Nuphar lutea (L.) S. et .S., Nymphaea alba L., Anacamptis pyramidalis (L.) Rich. and Himantoglossum hircinum (L.) Spreng, according to Bulgarian Biological Diversity Act, Appendix 3, Article 37. Conservation measures and regulated use is required for Leucojum aestivum L. and Orchis purpurea Huds as per the Bulgarian Biological Diversity Act, Appendix 4, Art. 41 (1). There are seven species prohibited for collection from their natural habitats pursuant to Order No. RD-83 (03.02.14) of the Minister of Environment and Water issued on the grounds of the Medicinal Plants Act, Art. 10 (1, 2, 3): Althea officinalis L., Artemisia santonicum L. subsp. patens (Neibr.) K. Pers., Glaucium flavum Crantz, Inula helenium L., Orchis purpurea Huds., Ruscus aculeatus L. and Valeriana officinalis $\mathrm{L}$.

There is one medicinal plant under special protection and use regulations-Betonica officinalis L, for which maximum quantities for collection from its natural habitat are annually set. The above restriction is set by Order No. RD-83 of 03.02.2014 of the Minister for the Environment and Water issued on the basis of the Medicinal Plants Act, Article 10 $(1,2,3)$.

We grouped the established for Kamchia Nature Complex medicinal plants according to the diseases they are applicable for in Table 1.

Analysis suggests more than 30 different types of healing activities of the established medicinal plants. Most of them act as diuretics (18 species). This number does not exceed significantly the number of other groups with different healing activities. The predominant part of species (59), representing one 
Biodiversity and Healing Activities of Medicinal Plants in the Area of Kamchia Nature Complex

Table 1. Groups of diseases, healing activities and plant substance

Plant Substance

\section{Plants Used for Treatment of Cardiovascular Diseases}

\begin{tabular}{|c|c|c|}
\hline Allium rotundum $\mathrm{L}$. & atherosclerosis, antimicrobial & Bulbus Allii \\
\hline Crataegus monogyna Jacq. & $\begin{array}{l}\text { cardiovascular, decreasing blood } \\
\text { pressure, sedative }\end{array}$ & Folium et flos Crataegi cum foliis \\
\hline Geranium robertianum $L$. & hypotensive, sedative & Rhizoma, folim et flos Geranii \\
\hline Lycopus europaeus L. & cardiovascular & Herba Lycopi \\
\hline Nymphaea alba L. & cardioactive agent & Rhizoma Nymphaeae albae \\
\hline Pastinaca sativa $\mathrm{L}$. & $\begin{array}{l}\text { cardiovascular, spasmolytic, } \\
\text { hypotensive }\end{array}$ & Radix et fructus Pastinacae \\
\hline Periploca graeca L. & cardioactiv agent & $\begin{array}{l}\text { Cortex seu stipites Periplocae } \\
\text { graecae }\end{array}$ \\
\hline Thalictrum flavum L. & hypotensive, antitumor action & Herba Thalictri \\
\hline Viscum album L. & $\begin{array}{l}\text { hypotensive, cardiotonic agent, } \\
\text { vasodilating }\end{array}$ & Herba Visci \\
\hline \multicolumn{3}{|c|}{ Plants Used for Treatment of Gastrointestinal Diseases } \\
\hline Alnus glutinosa (L.) Gaertn. & antidiarrheal agent, astringent & Fructus, folium et cortex Alni \\
\hline Artemisia vulgaris $\mathrm{L}$. & $\begin{array}{l}\text { appetite exciting, sedative, } \\
\text { haemostatic action }\end{array}$ & Herba et radix Artemisiae \\
\hline Arum maculatum L. & anti-inflammatory & Tubura Ari \\
\hline Ballota nigra L. & $\begin{array}{l}\text { spasmolytic, anti-inflammatory, pain } \\
\text { reliever }\end{array}$ & Herba Ballotae \\
\hline Betonica officinalis L. & spasmolytic, stimulates the appetite & Rizoma, radix et herba Betonicae \\
\hline Carpinus betulus L. & antimicrobial, antidiarrheal agent & Folium, flos et cortex Carpini \\
\hline Centaurea cyanus L. & $\begin{array}{l}\text { appetite exciting, stimulates the } \\
\text { release of bile, diuretic }\end{array}$ & Flores Centaureae \\
\hline Chamomilla recutita (L.) Rausch & $\begin{array}{l}\text { anti-inflammatory, antiseptic, } \\
\text { spasmolytic }\end{array}$ & Flores Chamomillae \\
\hline Chelidonium majus L. & $\begin{array}{l}\text { spasmolytic, stimulates the release } \\
\text { of bile }\end{array}$ & Herba Chelidonii \\
\hline Convolvulus arvensis L. & laxative, diuretic, epithelium tonic & Herba Convolvuli \\
\hline Cornus mas L. & astringent & Fructus Corni \\
\hline Cuscuta europaea L. & purgative, diuretic, pain reliever & Herba Cuscutae \\
\hline Datura stramonium L. & spasmolytic & Folium Stramonii \\
\hline Frangula alnus Mill. & laxative, antidiarrheal agent & Cortex Frangulae \\
\hline Fraxinus ornus L. & astringent & Cortex Fraxini \\
\hline Fraxinus oxycarpa Wild. & astringent & Cortex Fraxini \\
\hline
\end{tabular}


Djeni Cherneva, Dobri Ivanov

\begin{tabular}{|c|c|c|}
\hline Fumaria officinalis L. & $\begin{array}{l}\text { spasmolytic, stimulates the release } \\
\text { of bile }\end{array}$ & Herba Fumariae \\
\hline Geum urbanum L. & $\begin{array}{l}\text { anti-inflammatory, antidiarrheal } \\
\text { agent, antimicrobial }\end{array}$ & Rhizoma et radix Gei urbani \\
\hline Heracleum sibiricum L. & spasmolytic, hypotensive & Radix et fructus Heraclei sibirici \\
\hline Lotus corniculatus L. & spasmolytic, analgesic & Herba Loti corniculati \\
\hline Malus sylvestris Mill. & astringent, hypotensive, & Fructus Mali sylvestris \\
\hline Malva sylvestris L. & $\begin{array}{l}\text { spasmolytic, expectorant, } \\
\text { expectorant,sedative }\end{array}$ & Flos et folium Malvae sylvestris \\
\hline Nuphar lutea (L.) S. et .S. & anti-inflammatory & Rhizoma Nupharis lutei \\
\hline Prunus spinosa L. & astringent, anti-inflammatory & Flos et fructus Pruni spinosae \\
\hline Pulicaria dysentherica (L.) Bernh. & laxative, against insects & Herba et radux Pulicariae \\
\hline Rubus caesius L. & $\begin{array}{l}\text { astringent, antidiarrheal agent, anti- } \\
\text { inflammatory }\end{array}$ & $\begin{array}{l}\text { Radix folium et fructus Rubus } \\
\text { fruticosi }\end{array}$ \\
\hline Solanum nigrum L. & spasmolytic, sedative, anesthetic & Herba Solani nigri \\
\hline Teucrium polium L. & $\begin{array}{l}\text { constipative, haemostatic action, } \\
\text { disinfecting }\end{array}$ & Herba Teucrii \\
\hline Teucrium chamaedrys L. & $\begin{array}{l}\text { anti-inflammatory, anesthetic, } \\
\text { astringent, antidiarrheal agent }\end{array}$ & Herba Teucrii \\
\hline Ulmus minor Mill. & $\begin{array}{l}\text { astringent, antidiarrheal agent, anti- } \\
\text { inflammatory }\end{array}$ & Cortex Ulmi \\
\hline \multicolumn{3}{|c|}{ Plants Used for Treatment of the Liver and Biliary Tract } \\
\hline Cichorium inthybus L. & $\begin{array}{l}\text { appetite exciting, diuretic, stimulates } \\
\text { the release of bile }\end{array}$ & Radix Cichorii \\
\hline $\begin{array}{l}\text { Mentha } \\
\text { aquatica L. }\end{array}$ & spasmolytic, carminative, antiseptic & Folium Menthae aquaticae \\
\hline Mentha pulegium L. & spasmolytic, carminative, antiseptic & Folium Menthae pulegiumae \\
\hline Rumex crispus L. & stimulates the release of bile, laxative, & Radix et folium Rumicis crispis \\
\hline Rumex acetosella L. & stimulates the release of bile, laxative & Radix et folium Rumicis acetosellis \\
\hline Rumex hydrolapathum Huds. & stimulates the release of bile, laxative & $\begin{array}{l}\text { Radix et folium Rumicis } \\
\text { hydropalathumis }\end{array}$ \\
\hline Taraxacum officinalis Veb. & stimulates the release of bile, diuretic & Herba et Radix Taraxaci \\
\hline \multicolumn{3}{|c|}{ Plants Used for Treatment of Respiratory Diseases } \\
\hline Althea officinalis L. & expectorant, anti-inflammatory & Radix Althaeae \\
\hline Anacamptis pyramidalis (L.) Rich. & expectorant, anti-inflammatory & Tuber Salep \\
\hline Angelica sylvestris L. & $\begin{array}{l}\text { expectorant, stimulates sweating, } \\
\text { spasmolytic, diuretic }\end{array}$ & Rhizoma et radix Angelicae sylvestris \\
\hline Glaucium flavum Crantz & cough suppressant & Herba Glauci flavi \\
\hline
\end{tabular}


Biodiversity and Healing Activities of Medicinal Plants in the Area of Kamchia Nature Complex

\begin{tabular}{|c|c|c|}
\hline Hedera helix L. & $\begin{array}{l}\text { anti-inflammatory, expectorant, } \\
\text { broncholytic }\end{array}$ & Folium Hedere helicis \\
\hline $\begin{array}{l}\text { Himantoglossum hircinum (L.) } \\
\text { Spreng. }\end{array}$ & expectorant, anti-inflammatory & Tuber Salep \\
\hline Inula helenium L. & $\begin{array}{l}\text { anti-inflammatory, expectorant, } \\
\text { anthelmintic }\end{array}$ & Radix Inulae helenii \\
\hline Iris pseudacorus L. & $\begin{array}{l}\text { expectorant, anti-inflammatory, } \\
\text { analgetic }\end{array}$ & Rizoma Iridis \\
\hline Iris pumila L. & anti-inflammatory & Rhizoma Iridis \\
\hline Lysimachia nummularia L. & expectorant, diuretic & Radix et flos Primulae \\
\hline Orchis purpurea Huds. & expectorant, anti-inflammatory & Tuber Salep \\
\hline Paliurus spina-christi Mill. & $\begin{array}{l}\text { expectorant, anti-inflammatory, } \\
\text { spasmolytic }\end{array}$ & Fructus Paliuri \\
\hline Papaver rhoeas L. & expectorant & Flos Rhoeados \\
\hline Platanthera bifolia Rich. & expectorant, anti-inflammatory & Tuber Salep \\
\hline Primula acaulis (L.) Grubb. & expectorant, diuretic, sedative & Radix et flos Primulae \\
\hline Pulmonaria officinalis L. & expectorant, anti-inflammatory & Herba Pulmonariae \\
\hline Saponaria officinalis L. & $\begin{array}{l}\text { expectorant, diuretic, stimulation of } \\
\text { sweat }\end{array}$ & Radix Saponariae rubrae \\
\hline Senecio jacobaea L. & spasmolytic, antiasthmatic & Rhizoma et Herba \\
\hline Siderites montana L. & expectorant & Herba Sideritis montanae \\
\hline Sisymbrium officinale (L.) Scop. & expectorant, diuretic, & Herba Sisymbrili \\
\hline Trifolium arvense $\mathrm{L}$. & $\begin{array}{l}\text { expectorant, haemostatic action, } \\
\text { diuretic, anti-inflammatory }\end{array}$ & Herba et flos Trifolii arvensis \\
\hline Trifolium pratense L. & $\begin{array}{l}\text { expectorant, haemostatic action, } \\
\text { diuretic, anti-inflammatory }\end{array}$ & Herba et flos Trifolii pratensis \\
\hline Verbascum phlomoides L. & expectorant, anti-inflammatory & Flos Verbasci \\
\hline Verbascum phoeniceum L. & $\begin{array}{l}\text { expectorant, expectorant, anti- } \\
\text { inflammatory }\end{array}$ & Flos Verbasci \\
\hline Veronica anagalis-aquatica L. & $\begin{array}{l}\text { broncholytic, expectorant, anti- } \\
\text { inflammatory }\end{array}$ & Herba Veronicae \\
\hline Veronica arvensis $\mathrm{L}$. & expectorant, anti-inflammatory & Herba Veronicae \\
\hline $\begin{array}{l}\text { Veronica austriaca L.subsp. jacquinii } \\
\text { (Baumg.) Maly }\end{array}$ & $\begin{array}{l}\text { broncholytic, expectorant, anti- } \\
\text { inflammatory }\end{array}$ & Herba Veronicae \\
\hline Veronica beccabunga L. & $\begin{array}{l}\text { broncholytic, expectorant, anti- } \\
\text { inflammatory }\end{array}$ & Herba Veronicae \\
\hline Viola odorata L. & expectorant, diuretic & Radix, rhizoma, herba et flos Violae \\
\hline \multicolumn{3}{|c|}{ Plants Used for Treatment of Kidney and Urinary Tract Diseases } \\
\hline Alisma plantago-aquatica L. & diuretic & Rhizoma Plantaginis aquaticae \\
\hline
\end{tabular}


Djeni Cherneva, Dobri Ivanov

\begin{tabular}{|c|c|c|}
\hline Anagalis arvensis L. & $\begin{array}{l}\text { diuretic, expectorant, anti- } \\
\text { inflammatory }\end{array}$ & Herba Anagallidis \\
\hline Arctium lappa L. & diuretic, anti-ulcer & Radix Bardanae \\
\hline Arctium tomentosum Mill. & diuretic, anti-ulcer & Radix Bardanae \\
\hline Asperula odorata $\mathrm{L}$. & $\begin{array}{l}\text { diuretic, stimulation of sweat, } \\
\text { expectorant }\end{array}$ & Herba Asperulae \\
\hline Astragalus glycyphyllos L. & $\begin{array}{l}\text { diuretic, anti-inflammatory, } \\
\text { antihypertensive }\end{array}$ & Herba Astragali glucyphylli \\
\hline Cynodon dactylon L. & diuretic, laxative & Rhizoma Graminis italici \\
\hline Eryngium campestre L. & diuretic, spasmolytic & Radix Eringii \\
\hline Eryngium maritimum L. & diuretic, spasmolytic & Radix Eringii \\
\hline Fragaria vesca L. & $\begin{array}{l}\text { diuretic, anti-inflammatory, anti } \\
\text { atherosclerosis activity }\end{array}$ & Fructus et folium Fragariae \\
\hline Galium palustre L. & $\begin{array}{l}\text { astringent, anti-inflammatory, } \\
\text { antimicrobial, laxative }\end{array}$ & Herba Galii palustri \\
\hline Galium aparine $\mathrm{L}$. & diuretic, laxative, pain reliever & Herba Galii aparinis \\
\hline Oenanthe aquatica (L.) Poir. & diuretic, spasmolytic, expectorant & Fructus Phellandrii \\
\hline Ononis spinosa L. & diuretic, anti-inflammatory & Radix Ononidis \\
\hline Physalis alkekengii $\mathrm{L}$. & diuretic, anti-inflammatory & Fructus Alkekengi \\
\hline Polygonum aviculare L. & $\begin{array}{l}\text { diuretic, astringent, haemostatic } \\
\text { action }\end{array}$ & Herba Polygoni avicularis \\
\hline Populus tremula L. & diuretic, antiseptic & Gemma Populi \\
\hline Populus nigra L. & diuretic, antiseptic & Gemma Populi \\
\hline Prunella vulgaris L. & pain reliever, diuretic & Herba Prunellae vulgaris \\
\hline Ruscus aculeatus L. & diuretic, astringent, antihemorrhoid & Rhizoma et radix Rusci \\
\hline Sambucus ebulus L. & diuretic, antiseptic, expectorant & Radix,fructus et flos Ebuli \\
\hline \multicolumn{3}{|c|}{ Plants Used for Treatment of Rheumatic Diseases and Colds } \\
\hline Colchicum autumnale L. & antitumor action, pain reliever & Bulbo-tuber Colchici \\
\hline Filipendula vulgaris Moench & anti-rheumatic, diuretic & Herba Filipendulae \\
\hline Phytolaca americana L. & $\begin{array}{l}\text { anti-inflammatory, anti-rheumatic, } \\
\text { laxative }\end{array}$ & Radix et folium Phytolaccae \\
\hline Salix alba L. & antipyretic, anti-rheumatic & Cortex Salicis \\
\hline Sambucus nigra L. & stimulation of sweat, diuretic & Flores et fructus Sambuci \\
\hline Sambucus racemosa $\mathrm{L}$. & $\begin{array}{l}\text { anti-inflammatory, antioxidant } \\
\text { action }\end{array}$ & Radix et Fructus Sambuci \\
\hline Sinapis arvensis $\mathrm{L}$. & skin-warming action & Semen Sinapis arvensae \\
\hline Smilax excelsa $\mathrm{L}$. & influenza, antipyretic & Herba Smilax exelsi \\
\hline Solanum dulcamara L. & $\begin{array}{l}\text { stimulation of sweat, anti- } \\
\text { inflammatory, diuretic, laxative }\end{array}$ & Herba Dulcamarae \\
\hline Sorbus torminalis (L.) Crantz & anti-rheumatic, astringent, diuretic & Fructus Sorbi torminalae \\
\hline
\end{tabular}


Biodiversity and Healing Activities of Medicinal Plants in the Area of Kamchia Nature Complex

\begin{tabular}{|c|c|c|}
\hline Verbena officinalis L. & $\begin{array}{l}\text { stimulation of sweat, antipyretic, } \\
\text { sedative }\end{array}$ & Herba Verbenae \\
\hline Xanthium spinosum L. & anti-rheumatic, anti-inflammatory & Herba et fructus Xanthii spinosi \\
\hline Xanthium strumarium L. & anti-rheumatic, anti-inflammatory & Herba et fructus Xanthii strumarii \\
\hline \multicolumn{3}{|c|}{ Plants Used for Treatment of Metabolic and Endocrine Diseases } \\
\hline Galega officinalis L. & hypoglycaemic, diuretic & Herba Galegae \\
\hline Lemna minor $\mathrm{L}$. & $\begin{array}{l}\text { antipyretic, anti-inflammatory, } \\
\text { stimulates the release of bile, }\end{array}$ & Herba Lemnae \\
\hline Lepidium ruderale L. & $\begin{array}{l}\text { antidiabetic, stimulation of sweat, } \\
\text { diuretic, sedative }\end{array}$ & Herba Lepidii \\
\hline Xeranthemum annuum L. & $\begin{array}{l}\text { antiviral action, antibacterial, } \\
\text { antimycotic, strengthens the } \\
\text { immune system }\end{array}$ & Herba Xeranthemii \\
\hline \multicolumn{3}{|c|}{ Plants Used for Treatment of Parasitic Diseases } \\
\hline Artemisia campestris L. & anthelmintic & Herba Artemisiae \\
\hline $\begin{array}{l}\text { Artemisia santonicum L. subsp. } \\
\text { patens (Neibr.) K.Pers. }\end{array}$ & anthelmintic & Herba et radix Artemisiae \\
\hline Daucus carota L. & anthelmintic, source of vitamin A & Radix et semen Dauci \\
\hline Pteridium aquilinum (L.) Kuhn. & anthelmintic & Rhizoma et folium Aquilinae \\
\hline Tanacetum vulgare $\mathrm{L}$. & anthelmintic, antiseptic, spasmolytic & Herba Tanaceti vulgare \\
\hline \multicolumn{3}{|c|}{ Plants That Affect the Central Nervous System } \\
\hline Conium maculatum L. & pain reliever & Fructus et Herba Conii \\
\hline Consolida regalis S. F. Gray & curare-like action & Herba et semen Consolidae \\
\hline Humulus lupulus L. & sedative & Strobuli Lupuli \\
\hline Leucojum aestivum $\mathrm{L}$. & $\begin{array}{l}\text { improves neuromuscular } \\
\text { conduction, curare-like action }\end{array}$ & Herba Leucoji aestivi \\
\hline Melilotus alba Med. & sedative & Herba Meliloti \\
\hline Melilotus officinalis (L.) Pall. & sedative & Herba Meliloti \\
\hline Scutellaria altissima $\mathrm{L}$. & $\begin{array}{l}\text { spasmolytic, astringent, diuretic, } \\
\text { sedative }\end{array}$ & Herba Scutelarii \\
\hline Valeriana officinalis L. & sedative, spasmolytic, hypotensive & Radix et rhizoma Valerianae \\
\hline \multicolumn{3}{|c|}{ Plants with a Predominantly Haemostatic Action } \\
\hline Acer tataricum $\mathrm{L}$. & astringent, anti-inflammatory & Folium Aceri tatarici \\
\hline Bidens tripartita L. & $\begin{array}{l}\text { astringent, diuretic, stimulation of } \\
\text { sweat, expectorant }\end{array}$ & Herba Bidentis \\
\hline Capsella bursa-pastoris (L.) Medicus. & haemostatic action & Herba Bursae - pastoris \\
\hline Erodium cicutarium (L.) L Her. & haemostatic action & Herba Erodii cicutarii \\
\hline Loranthus europaeus L. & antihemorrhoidal & Herba Loranthi \\
\hline
\end{tabular}


Djeni Cherneva, Dobri Ivanov

\begin{tabular}{|c|c|c|}
\hline Lythrum salicaria L. & $\begin{array}{l}\text { astringent, haemostatic action, } \\
\text { antiseptic, antidiarrheal agent }\end{array}$ & Herba Salicariae \\
\hline Lythrum virgatum $\mathrm{L}$. & $\begin{array}{l}\text { astringent, haemostatic action, } \\
\text { antiseptic, antidiarrheal agent }\end{array}$ & Herba Salicariae \\
\hline Persicaria hydropiper (L.) Opiz. & haemostatic action & Herba Polygoni hydropiperis \\
\hline Persicaria maculata (Raf.) S.F.Gray & haemostatic action, & Herba Poligonii hydropiperis \\
\hline Quercus cerris L. & $\begin{array}{l}\text { astringent, haemostatic action, anti- } \\
\text { inflammatory }\end{array}$ & Cortex et fructus Quercus \\
\hline Quercus frainetto Ten. & $\begin{array}{l}\text { astringent, haemostatic action, anti- } \\
\text { inflammatory }\end{array}$ & Cortex et fructus Quercus \\
\hline Sangusorba minor Scop. & $\begin{array}{l}\text { haemostatic action, astringent, anti- } \\
\text { inflammatory, constipative }\end{array}$ & Rhizona et radix Sanguisorbe \\
\hline Sanquisorba officinalis L. & $\begin{array}{l}\text { haemostatic action, astringent, anti- } \\
\text { inflammatory, constipative }\end{array}$ & Rhizona et radix Sanguisorbe \\
\hline Urtica dioica L. & haemostatic action, diuretic & Folium Urticae \\
\hline Viburnum opulus L. & haemostatic action, sedative & Cortex et extractum Viburni fluidum \\
\hline Aristolochia clematitis L. & wound healing & $\begin{array}{l}\text { Radix, rizoma et herba Aristolochiae } \\
\text { clematitis }\end{array}$ \\
\hline Caltha palustris L. & anti-inflammatory, anesthetic & Herba Calthae palustris \\
\hline Hypericum perforatum L. & $\begin{array}{l}\text { anti-inflammatory, astringent, anti- } \\
\text { ulcer, haemostatic action, sedative, } \\
\text { wound healing }\end{array}$ & Herba Hyperici \\
\hline Plantago major L. & $\begin{array}{l}\text { anti-inflammatory, expectorant, } \\
\text { laxative, anti-ulcer, diuretic }\end{array}$ & Folium et herba Plantaginis majoris \\
\hline Plantago arenaria $\mathrm{W}$. et $\mathrm{K}$. & $\begin{array}{l}\text { anti-inflammatory, expectorant, } \\
\text { anti-ulcer, }\end{array}$ & $\begin{array}{l}\text { Folium et herba Plantaginis } \\
\text { arenariae }\end{array}$ \\
\hline Plantago lanceolata L. & $\begin{array}{l}\text { anti-inflammatory, expectorant, } \\
\text { anti-ulcer }\end{array}$ & $\begin{array}{l}\text { Folium et herba Plantaginis } \\
\text { lanceolatae }\end{array}$ \\
\hline Stachys recta $\mathrm{L}$. & regenerative, antispastic & Herba Stachi rectae \\
\hline Symphytum officinale L. & wound healing, anti-ulcer & Radix Symphyti \\
\hline \multicolumn{3}{|c|}{ Plants Used in Skin Diseases } \\
\hline Bellis perennis L. & wound healing, expectorant, & Flores Bellidis perennis \\
\hline Clematis vitalba $\mathrm{L}$. & $\begin{array}{l}\text { anti-inflammatory, antimicrobial, } \\
\text { wound healing }\end{array}$ & $\begin{array}{l}\text { Radix folium et flos Clematidis } \\
\text { vitalbae }\end{array}$ \\
\hline Euphorbia amygdaloides L. & keratolytic & Succus Euphorbiae \\
\hline Euphorbia myrsinites L. & keratolytic & Succus Euphorbiae \\
\hline Fagus orientalis Lipsky & antiseptic, antimycotic & Pix, Fructus et folium \\
\hline \multicolumn{3}{|c|}{ Plants with Other Types of Actions } \\
\hline Anthemis tinctoria $\mathrm{L}$. & hair bleaching & $\begin{array}{l}\text { Fructus, folium et cortex Anthemis } \\
\text { tinctorii }\end{array}$ \\
\hline Butomus umbellatum L. & nutrient & Rizoma Butomi \\
\hline
\end{tabular}


Biodiversity and Healing Activities of Medicinal Plants in the Area of Kamchia Nature Complex

\begin{tabular}{|l|l|l|}
\hline Equisetum palustre L. & - & Herba Equiseti \\
\hline Lamium purpureum L. & nutrient, nectariferous & Herba Lamii \\
\hline Lathyrus niger (L.) Bernh. & fodder, nectariferous & Herba Lathyri nigri \\
\hline Lathyrus pratensis L. & fodder, nectariferous & Herba Lathiri pratensi \\
\hline Ranunculus ficaria L. & antibacterial action & Herba et Rhizoma Ficarii \\
\hline Ranunculus repens L. & pain reliever & $\begin{array}{l}\text { Herba et Rhizoma Ranunculi } \\
\text { repensis }\end{array}$ \\
\hline Rosa corymbifera Borkh. & $\begin{array}{l}\text { rich in vitamins, preventing } \\
\text { scorching, astringent, diuretic }\end{array}$ & Fructus Rosae \\
\hline Trifolium repens L. & fodder & Herba Trifolii repensis \\
\hline Vicia grandiflora Scop. & fodder & Herba Vicii \\
\hline
\end{tabular}

fifth of the featured medicinal plants, are used mainly for treatment of gastrointestinal diseases and respiratory diseases.

Different morphological and generative parts of the established medicinal plants are used as plant substances. The species in which the above ground portion of the plant is collected for its plant substance dominate the rest and constitute half of the established for the area medicinal plants. One third of the species can be collected and used for different plant parts.

\section{CONCLUSION}

Survey results reveal a considerable variety of medicinal plants in Kamchia Natural Complex area. They represent an integral part of the country's resource of medicinal plants. Their presence enhances and highlights floodplain forest's significance, manifests the uniqueness of coastal sands and dunes as habitats with priority conservation status. The established medicinal plants feature a variety of healing activities and are applicable for a wide range of diseases. Findings suggest further resource-based research in view of their protection and rational use.

\section{REFERENCES}

1. Michev T. Etat general des zones humides en Bulgarie. In: Michev T, editor. Plan National d'actions prioritaires de conservation des zones humides les plus importantes de Bulgarie. Ministere de l'environment de Bulgarie et Bureau de la Convention de Ramsar. Sofie: 1993. pp. 9-10.

2. Michev T, Stoyneva M. Conservation of Bulgarian non-lotic wetlands. In: Michev, T, Stoyneva M, editors. Inventory of Bulgarian Wetlands and their Biodiversity. Sofia: 2007.

3. Ivanov D, Filipova-Marinova M, Dimitrov D. Flora and vegetation of the nature complex „Kamchia“. Proceedings of the National Museum, Varna. 2002; 32-33(47-48): 314-40. (In Bulgarian).

4. Ivanov D, Filipova-Marinova M. Medicinal plants of wetlands along the Northern Black Sea coast. Hist Med Coll; 2008; 4-5:50-7. (In Bulgarian).

5. Zahariev D, Boycheva P, Kosev K. Review on the medicinal plants of the north black sea coast (Bulgaria). Annual of Sofia University, Faculty of Biology. 2016; 99(2):100-14.

6. Tolmachev AI. Introduction to plant geography. Publishing house of the Leningrad University; 1974. (In Russian).

7. Jordanov D, editor. Florae Reipublicae Popularis Bulgaricae. Vol. I-IX. Serdicae: Aedibus Acad. Sci. Bulgaricae; 1963-1989. (In Bulgarian).

8. Velchev V, editor. Florae Reipublicae Popularis Bulgaricae. Vol. 8. Serdicae: Aedibus Acad. Sci. Bulgaricae; 1982. p. 518. (In Bulgarian).

9. Velchev V, editor. Florae Reipublicae Popularis Bulgaricae. Vol. 9. Serdicae: Aedibus Acad. Sci. Bulgaricae; 1989. p. 539. (In Bulgarian).

10. Kozhuharov S, editor. Flora of the Republic of Bulgaria. Vol. X-XI. Sofia: Prof. M. Drinov Acad. Publ; 1995. p. 428. (In Bulgarian).

11. Peev D, editor. 2013. Flora of the Republic of Bulgaria. Vol. XI, Sofia: Prof. M. Drinov Acad. Publ; 2013. p. 523. (In Bulgarian).

12. Kozhuharov S, editor, Andreev N, Anchev M, Kozhuharov S, Markova M, Peev D, et al. Determi- 
nant of the vascular plants in Bulgaria. Sofia: Science and Art; 1992.

13. Assyov B, Petrova A, Dimitrov D, Vassilev R. Conspectus of the Bulgarian vascular flora. Distribution maps and floristic elements. Sofia: Bulgarian Biodiversity Foundation; 2006. p. 452. (In Bulgarian).

14. Medicinal Plants Act of the Republic of Bulgaria. Annex. State Gazette. 2000;29:9-29. Last amended in: State Gazette. 2014; 98. (In Bulgarian).

15. Hardalova R, Evstatieva L, Gusev C. Characteristics of the resource from the wonderfully healing plants in Bulgaria and the provision for sustainable development. In: National strategy behind bio-diversity denomination. 1994; 2:41-72. (In Bulgarian).

16. Peev D, editor. Red Data Book of the Republic of Bulgaria. Vol. 1. Plants and fungi. Available at: http://e-ecodb.bas.bg/rdb/bg/voll/Lycinund=html (accessed on 01.08.2013).

17. Law on Biodiversity of the Republic of Bulgaria. Annex. State Gazette. 2002;77. Last amended in State Gazette. 2017; 76. (In Bulgarian).

18. Order NoRD-83 from 3 February 2014 for special arrangements for the conservation and use of medicinal plants. State Gazette. 2014; 14. (In Bulgarian).
19. Lukas G. List of rare threatened and endemic plants in Europe. Strasbourg: Council of Europe; 1983.

20. The IUCN Red List of threatened species. 2014.3.

21. Bern Convention, 1979: Convention on the Conservation of European Wildlife and Natural Habitats. Appendix I (enforced in Bulgaria on 01.05.1991)

22. CITES, 1975: Convention on International Trade in Endangered Species of Wild Fauna and Flora.

23. Petrova A, Vladimirov V. Balkan endemics in the Bulgarian flora. Sofia: Phytologia Balcanica. 2010; 16(2):293-311.

24. Petrova A, Velchev V. List of Bulgarian endemic species. In: Petrova A, editor. Atlas of Bulgarian Endemic Plants. Sofia: Gea-Libris Ltd.; 2006; p. 399.

25. Petkov V, editor. Contemporary phytotherapy. Sofia: Medicine and Physical Education; 1982. (In Bulgarian).

26. Asenov I, Nikolov S. Pharmacognosy. Sofia: Medicine and Physical Education; 1988. (In Bulgarian).

27. Nikolov S, editor. Specialized encyclopedia of medicinal plants in Bulgaria. Sofia: Trud; 2006. p. 566. (In Bulgarian). 